\title{
The Results Observation on Adjuvant Chemotherapy with Microwave Hyperthermia and Stereotactic Radiotherapy in Retreated Elderly Patients with Advanced (III/IV Stage) Lung Cancer
}

\author{
Xihao Yu' ${ }^{1}$ Xinping Li1 ${ }^{1}$ Guiyun Dong1, Wenyan Yu' ${ }^{2}$, Jianping Wang1, Ying Xiong1 \\ ${ }^{1}$ Department of Oncology of Liberation Army 85 Hospital, Shanghai, China \\ ${ }^{2}$ Basic Medical College of Shanghai Jiao Tong University, Shanghai, China \\ Email: vixih@tom.com
}

Received 14 January 2016; accepted 23 March 2016; published 30 March 2016

\section{Abstract}

Objective: To evaluate the significance of adjuvant chemotherapy and radiotherapy (Gamma-knife) in the elderly patients with retreated advance lung cancer. Methods 83 patients (age 50 to 81 years) with retreating advanced (III/IV stage) lung cancer were divided into three groups according to patients received treating by different methods. The cases were treated by synchronous chemo-radiotherapy (A group), sequentially chemo-radiotherapy (B group) and continuous systematized chemotherapy alone ( $\mathrm{C}$ group). The systemic 3-dimensional orientation apparatus was used in radiotherapy. The chemotherapy regimen in combined radiotherapy was given by common first line regimen on lung cancer. The regimen of chemotherapy combated with whole body hyperthermia by using micro-wave on abodeman about 2 or 3 line was used in C group only. Results: The overall responses rates (RR) of $A, B$ and $C$ groups were $17.1 \%, 11.5 \%$ and $31.8 \%$, but it was non-significance difference $(P>0.05)$. Overall responses rates (RR) of III and IV stage groups were $18.2 \%$ and $20.0 \%$. The average overall survival time (OS) and median survival time (MST) of the patients treated by chemotherapy ( $C$ group) were highest about 14.2 and 9.3 months (respectively at $A$ group 6.7 and 6.0 months. $P<0.01)$, in which $B$ group was longer than A group $(P<$ 0.05 ), and $95.5 \%$ CI value was not overlap. The total survival time of postoperative patients was longer in the various groups by different treated than that in non-operations. Conclusion: The rates (RR) of III and IV stage groups were non-significance difference. The higher of ORR, OS and MST in a few patients of re-treated advanced lung cancer was presented in case with treated by continuous system chemotherapy. There were survival superiority in initial postoperative patients groups by different treated. It is a profitable and effect treatment that the pure reasonable systemic chemotherapy was used for elderly case with advanced retreated lung cancer. 


\section{Keywords}

\section{Elderly Patients, Chemotherapy, Combined Radiotherapy, Lung Cancer}

\section{Background}

The retreating to elder patients with advanced lung cancer is a problems that often needs to be faced in clinical practice. A number of studies have shown that appropriately chemotherapy or radiotherapy is better than the best supportive care. Due to the special physiological function of old patients, it was considered that how to choose the best treatment for the patients. Especially for patients treated again, at present this kind of research is not much. It is object about the paper to investigate the effect and significance of adjuvant chemotherapy with hyperthermia and radiotherapy (Gamma-knife) in the treatment of elderly patients with retreated advance lung cancer.

\section{Clinical Material and Methods}

Eighty-three patients, median age 67.3 year (50 to 81 years), with retreating advanced (III/IV stage) lung cancer were divided into three groups according to patients received treating by different methods. 35 cases were treated by synchronous chemo-radiotherapy (group A). 26 cases were treated by sequentially chemo-radiotherapy (group B) and 22 cases were treated by continuous systematized chemotherapy alone (group C) (see Table 1). All patients were selected for inclusion in study after the initial treatment stoped 2 - 3 months. The systemic 3-dimensional orientation apparatus was used in radiotherapy. The total dose of 35-55GY was given in synchronous or sequential chemo-radiotherapy. The chemotherapy regimen in combined radiotherapy was given by common first line regimen on lung cancer. (NSCLC: NVB25 mg $/ \mathrm{m}^{2} \mathrm{~d} 1,8$, GEM $0.8-1.0 / \mathrm{m}^{2} \mathrm{~d} 1,8$, DDP $40 \mathrm{mg}$ d1-3 or DDP20 mg d1-5. SCLC: CBP AUC = 5, VP16 0.1/ $\mathrm{m}^{2}$. q21d). The part chemotherapy combated with whole body hyperthermia by using micro-wave on abodeman in temperature $40^{\circ} \mathrm{C}-42^{\circ} \mathrm{C}$ for $1-2$ hours. The regimen of chemotherapy about 2 or 3 line was used in C group only. Drugs was used at Taxol (Squibb US, Co) $175 \mathrm{mg} / \mathrm{m}^{2}$ d1, or Docertacel (Aventis Pharma, SA, Co) $70-80 \mathrm{mg} / \mathrm{m}^{2}$, d1; Pemetrexed (Lilly France SAS, Co) $500 \mathrm{mg} / \mathrm{m}^{2}$, d1.). The responce rate was statistically based on RECIST (WHO) method.

Statistical analysis The categorical variables percent date were compared between the two groups by using the inpendent $X^{2}$ test. Continuous data were compared between two groups by using independent $t$ test. A two-tailed $P$ value of $<0.05$ was considered statistically significant. Survival was calculated by using the Kaplan-Meier method and compared by using the log-rank test. The Kaplan-meier curve was used to exhibit survival time.

\section{Results}

1) The overall responses rates (RR) of A, B and C groups were $17.1 \%, 11.5 \%$ and $31.8 \%$, but it was non-significance difference respectively at three group $(\mathrm{P}>0.05)$. The higher effective rate was presented in stage III case with synchronous chemo-radiotherapy (23.1\%), and in stage IV with continuous systematized chemotherapy alone (38.5\%), but overall responses rates (RR) of III/IV stage groups was non-significance difference (18.2\% and 20.0\%) (see Table 2).

Table 1. The general state of the patients with cancer on different treating groups.

\begin{tabular}{|c|c|c|c|c|c|c|c|c|}
\hline \multirow{2}{*}{ Groups } & \multirow{2}{*}{ Cases } & \multicolumn{2}{|c|}{ SEX } & \multirow{2}{*}{ Mid-age } & \multicolumn{2}{|c|}{ Stage (\%) } & \multicolumn{2}{|c|}{ PS (\%) } \\
\hline & & M & $\mathrm{F}$ & & III & IV & $\leq 2$ & $\geq 3$ \\
\hline group A & 35 & 23 & 12 & 54.6 & $13(37.1)$ & $22(62.8)^{*}$ & 25 (71.4) & $10\left(28.6^{*}\right)$ \\
\hline group B & 26 & 20 & 6 & 51.2 & $11(42.3)$ & 15 (57.7) & 16 (61.5) & $10(38.5)$ \\
\hline group C & 22 & 13 & 9 & 56.5 & 9 (40.9) & $14(63.7)^{*}$ & 14 (63.7) & 8 (36.3) \\
\hline
\end{tabular}

${ }^{*} \mathrm{P}>0.05$. 
2) The average overall survival time (OS) and median survival time (MST) of the patients treated by chemotherapy (C group) were highest about 14.2 and 9.3 months (respectively at A group 6.7 and 6.0 months. $\mathrm{P}<$ $0.01)$, in which $B$ group was longer than A group $(\mathrm{P}<0.05)$, and 95.5\% CI value was not overlap. The Kaplanmeier curve for survival time was showed (shown in Figure 1).

3) The total survival time of initial postoperative patients were longer in the various groups by different treated than that in non-operations (see Table 3).

Table 2. The contrast about respond rate (RR) in different treating groups.

\begin{tabular}{|c|c|c|c|c|c|c|}
\hline \multirow{2}{*}{ Group } & \multicolumn{2}{|c|}{ Stage III } & \multicolumn{2}{|c|}{ Stage IV } & \multirow{2}{*}{\multicolumn{2}{|c|}{ RR (\%) }} \\
\hline & $\mathrm{N}$ & RR (\%) & $\mathrm{N}$ & RR (\%) & & \\
\hline Syn.che-rad (A) & 13 & $3(23.1)$ & 22 & $3(13.6)^{\#}$ & $6 / 35$ & $(17.1)$ \\
\hline Seq.che-rad (B) & 11 & $1(9.1)$ & 15 & $2(13.3)$ & $3 / 26$ & $(11.5)$ \\
\hline \multirow[t]{2}{*}{ Chem.onl (C) } & 9 & $2(22.2)$ & 13 & $5(38.5)^{+}$ & $7 / 22$ & $(31.8)^{*}$ \\
\hline & 33 & $6(18.2)$ & 50 & $10(20.0)$ & $16 / 83$ & (19.3) \\
\hline
\end{tabular}

${ }^{*} X^{2}=1.87 . \mathrm{P}>0.05$ (contrast between $\mathrm{C}$ and B); ${ }^{+} X^{2}=2.86 . \mathrm{P}>0.05$ (contrast between $\mathrm{C}$ and $\mathrm{A}$ ); ${ }^{\sharp} X^{2}=0.51 \mathrm{P}>0.05$ (contrast between stage IV and III in A group).

Table 3. Survival time (months) on different treating groups with post-operation.

\begin{tabular}{ccccccc}
\hline & \multicolumn{3}{c}{ No operation } & \multicolumn{3}{c}{ Post-operation } \\
\cline { 2 - 7 } Content & $\mathrm{n}$ & $\bar{X}$ & MST & $\mathrm{n}$ & $\bar{X}$ & MST \\
\hline Syn.che-rad (A) & 30 & 6.5 & 5.0 & 5 & 19.2 & 13.4 \\
Seq.che-rad (B) & 20 & 9.9 & 7.0 & 6 & 17.0 & 15.0 \\
Chem.onl (C) & 17 & 10.9 & 9.0 & 5 & 25.2 & 15.0 \\
& 65 & 9.1 & 7.0 & 16 & 20.5 & $14.5^{*}$ \\
\hline
\end{tabular}

${ }^{*} t=2.79 . \mathrm{P}<0.01$ (post-ope.cont no ope. about MST).

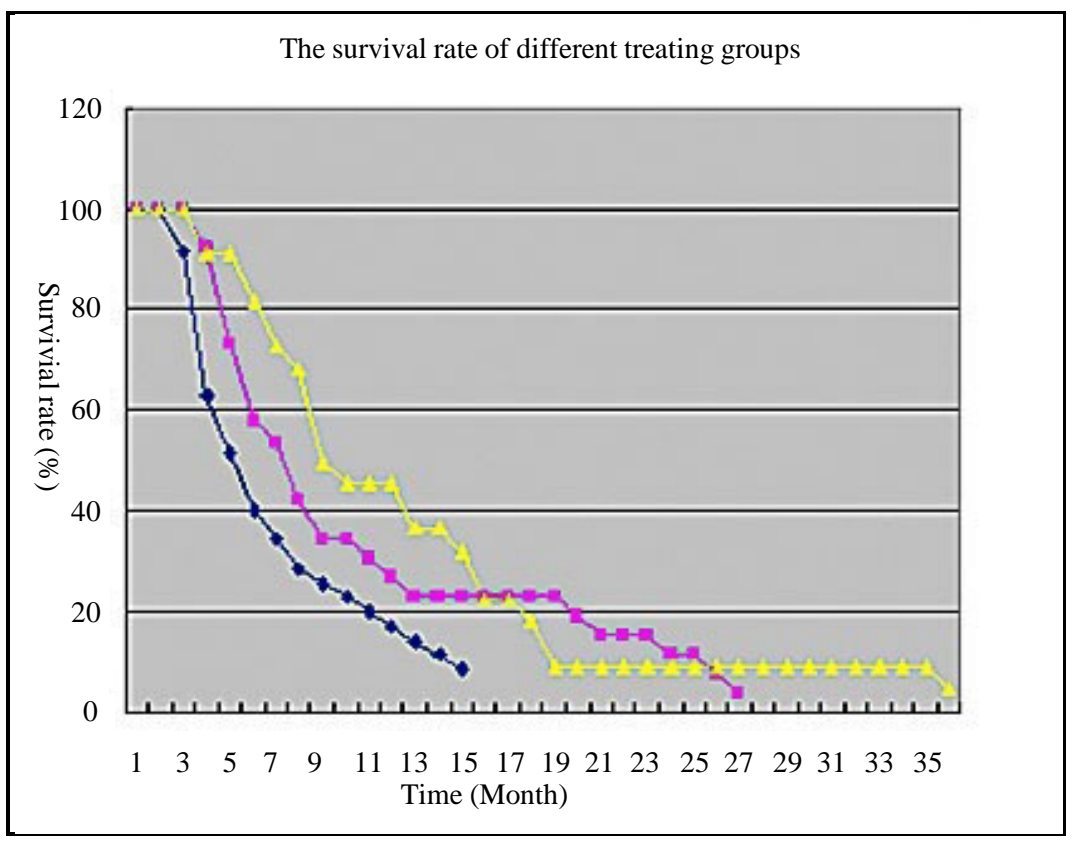

Figure 1. The Kaplan-meier survival rate of different treating groups. $-\bullet$ : group A, $-\mathbf{-}$ : group B, $-\mathbf{\Delta}$ : group C. 


\section{Discussion}

Stereotactic gamma knife radiotherapy for intracranial tumor and somatic localized tumors can make small part of radical effect to the operation as a knife. However, chemotherapy is often needed for patient with recurrence and metastasis [1]. However studies have indicated that, after radiotherapy or early chemotherapy, the residual tumorcells can accelerate the proliferation, which make the tumor more difficult to control [2]. So it is very important to follow up the system chemotherapy or consolidation chemotherapy. Hyperthermia can increase the efficacy of chemotherapy and reduce side effect, in which it can appropriately reduce the dose of chemotherapy drug and more suitable for the elderly. Although surgical treatment of lung cancer, especially in the initial treatment still occupies an important position [3], our research also shows that there were survival superiority in initial postoperative patients groups by subsequent different treated. But it is not possible for the elderly tumor patients to choose again. This clinical observation study of this paper suggested that the synchronous chemoradiotherapy can partly increased about RR of the patients with stage III case, and higher effective rate was presented in stage IV with continuous systematized chemotherapy, but the rates (RR) of III and IV stage groups were non-significance difference. The higher of ORR, OS and MST in a few patients of re-treated advanced lung cancer was presented in case with treated by continuous system chemotherapy. Conclusion: It is a profitable and effect treatment that the pure reasonable systemic chemotherapy was used for elderly case with advanced retreated lung cancer.

\section{References}

[1] Lin, J.-C., Jan, J.-S., Hsu, C.-Y., et al. (2003) Phase 3 Study of Concurrent Chemoradiotherapy versus Radiotherapy Alone for Advanced Nasopharyngeal Carcinoma: Positive Effect on Overall and Progression-Free Survival. Journal of Clinical Oncology, 21, 631-637. http://dx.doi.org/10.1200/JCO.2003.06.158

[2] Mehta, M., Scrimger, R., Mackkie, R., et al. (2001) A New Approach to Dose Escalation in Non-Small-Cell Lung Cancer. International Journal of Radiation Oncology Biology Physics, 49, $23-33$. http://dx.doi.org/10.1016/S0360-3016(00)01374-2

[3] Nagai, K., Sohara, Y., Tsachiya, R., et al. (2007) Prognosis of Resectd Non-Small Cell Lung Cancer Patients with Intrapalmonary Metastases. Journal of Thoracic Oncology, 2, 282-286. http://dx.doi.org/10.1097/01.JTO.0000263709.15955.8a 